$\mathrm{J}$ o u r n a l of

Mathematics

and Applications

JMA No 37, pp 85-96 (2014)

\title{
On efficient evaluation of integrals entering boundary equations of 3D potential and elasticity theory
}

\author{
Liliana Rybarska-Rusinek, Dawid Jaworski, \\ Aleksandr Linkov
}

\begin{abstract}
The paper presents recurrent formulae for efficient evaluation of all the integrals needed for solving static 3D potential and elasticity problems by the boundary elements method. The power-type asymptoties for the density at edges of the boundary are accounted for explicitly.
\end{abstract}

AMS Subject Classification: 65M38, 33E05

Keywords and Phrases: potential and elasticity problems, singular and hypersingular boundary integral equations, boundary element method, higher order approximations, edge element, elliptic integrals

\section{Introduction}

The purpose of the paper is to suggest an efficient general method for evaluation of the influence coefficients of the 3D boundary element method accounting for both smooth behaviour of the densities at internal parts of the boundary and power-type asymptotic behaviour near edges of the boundary.

Inspection of the boundary integrals equations of static 3D potential and elasticity theory [4] shows that it is sufficient to consider the function

$$
\int_{S^{q}} \frac{f(y)}{R} d S_{y},
$$

and its spatial derivatives $\partial / \partial x_{i}, \partial^{2} / \partial x_{i} \partial x_{j}, \partial^{3} / \partial x_{i} \partial x_{j} \partial x_{k}$.

Herein, $S^{q}$ is the surface of a boundary element; $f(y)$ is a function to be properly approximated on the element; $R=\sqrt{\left(x_{1}-y_{1}\right)^{2}+\left(x_{2}-y_{2}\right)^{2}+\left(x_{3}-y_{3}\right)^{2}}$, where $x_{1}$, $x_{2}, x_{3}$ and $y_{1}, y_{2}, y_{3}$ are global coordinates of the field and integration point, respectively.

COPYRIGHT (c) by Publishing Department Rzeszów University of Technology P.O. Box 85, 35-959 Rzeszów, Poland 


\section{Approximation of the boundary and density}

We shall assume that, as usual (e.g. [1]), a curvilinear, in general, surface element is transformed into a plane element. The global coordinates are transformed to the local Cartesian coordinates of the plane element with the local axes $y_{2}^{\prime}, y_{3}^{\prime}$ in the element plane; the origin $O^{\prime}$ is in the plane of the transformed element. Besides, we assume that the entries of Jacobian matrix, its determinant and the expression for $R$ are expanded into power series in $x_{i}^{\prime}-y_{i}^{\prime}$ and truncated. From now on, to simplify notation, we shall drop the prime in the transformed coordinates and refer (1.1) to a plane element in its local coordinates. Then $y_{1}=0$ and the function $f(y)$ is the product of the density depending on the local coordinates $y_{2}, y_{3}$ and powers of $y_{2}$ and $y_{3}$, which result from the truncated expressions mentioned.

Furthermore, we assume the plane element to be a trapezoid. This type of boundary elements includes as particular cases commonly used triangular, parallelogram, rectangular and square elements. Without loss of generality, we direct the $y_{2}$-axis along the trapezoid base, the $y_{3}$-axis orthogonal to it and we locate the origin in the lower left apex of the trapezoid (Fig. 1). For an edge element, we choose its edge as the base of the trapezoid. Then if the density near the edge has the power-type behaviour, it is described by the factor $y_{3}^{\alpha}$ with $0 \leqslant \alpha<1$. The general approximation of the function $f(y)$ in $(1.1)$ is of the form:

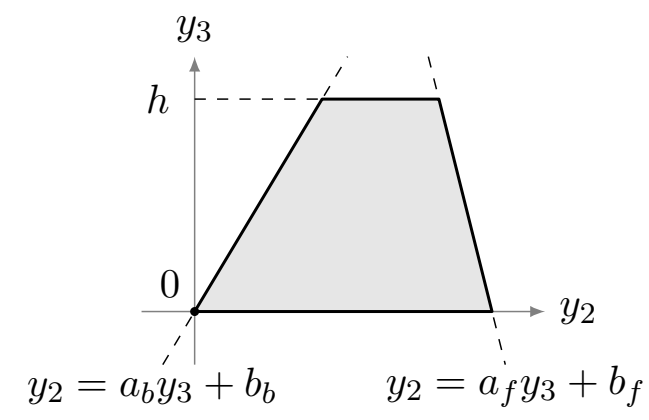

Figure 1: Trapezoidal element in local coordinates, $b_{b}=0$

$$
f(y)=y_{3}^{\alpha} \sum_{k+l=0}^{m_{p}} c_{k l} y_{2}^{k+s} y_{3}^{l+q}, \quad 0 \leqslant \alpha<1
$$

where $m_{p}$ is the degree of a polynomial approximating the density, $c_{k l}$ are coefficients of approximation, $s$ and $q$ are degrees arising from the coordinate transformation (for initially plane parts of the boundary $s=q=0$ ).

The two most important cases are: (i) $\alpha=0$ what corresponds to smooth behaviour of the density, and (ii) $\alpha=1 / 2$ what corresponds to square-root asymptotics typical for problem of linear fracture mechanics. Still, other exponents $\alpha$ may arise 
in approximations. For instance, $\alpha=2 / 3$ for fracturing impermeable rock by a Newtonian fluid. Therefore, it is reasonable to specify a particular value of $\alpha$ at the end of the discussion.

Using (2.1) in (1.1) with $S^{q}$ being the plane trapezoid of the height $h$ (Fig. 1 ) implies that it is sufficient to consider the main integrals of the form:

$$
A_{\alpha}^{k l}\left(x_{1}, x_{2}, x_{3}\right)=\int_{0}^{h}\left(y_{3}^{l+\alpha} \int_{y_{2}=a_{b} y_{3}+b_{b}}^{y_{2}=a_{f} y_{3}+b_{f}} \frac{\left(x_{2}-y_{2}\right)^{k}}{R} d y_{2}\right) d y_{3} .
$$

and their partial derivatives $\partial / \partial x_{i}, \partial^{2} / \partial x_{i} \partial x_{j}, \partial^{3} / \partial x_{i} \partial x_{j} \partial x_{k}$.

\section{Evaluation of the main integrals}

The integrals (2.2) are evaluated recurrently by using starting integrals for $k=0$ and $k=1$ :

$$
\begin{gathered}
A_{\alpha}^{0 j}\left(x_{1}, x_{2}, x_{3}\right)=-\int_{0}^{h}\left[y_{3}^{j+\alpha} \ln \left[\left(x_{2}-b_{\xi}-a_{\xi} y_{3}\right)+R_{\xi}\right]\right]_{\xi=b}^{\xi=f} d y_{3}, \\
A_{\alpha}^{1 j}\left(x_{1}, x_{2}, x_{3}\right)=-\int_{0}^{h}\left[y_{3}^{j+\alpha} R_{\xi}\right]_{\xi=b}^{\xi=f} d y_{3},
\end{gathered}
$$

where where $R_{\xi}=\sqrt{x_{1}^{2}+\left(x_{2}-b_{\xi}-a_{\xi} y_{3}\right)^{2}+\left(x_{3}-y_{3}\right)^{2}}$; the symbol []$_{x=a}^{x=b}$ means the double substitution: $[f(x)]_{x=a}^{x=b}=f(b)-f(a)$.

For $k \geqslant 2$, the recurrent equations are:

$$
\begin{gathered}
A_{\alpha}^{k l}=-\frac{1}{k}\left(\int_{0}^{h} y_{3}^{\alpha+l}\left[\left(x_{2}-b_{\xi}-a_{\xi} y_{3}\right)^{k-1} R_{\xi}\right]_{\xi=b}^{\xi=f} d y_{3}+\right. \\
\left.+(k-1)\left(x_{1}^{2}+x_{3}^{2}\right) A_{\alpha}^{(k-2) l}-2 x_{3}(k-1) A_{\alpha}^{(k-2)(l+1)}+(k-1) A_{\alpha}^{(k-2)(l+2)}\right) .
\end{gathered}
$$

Note that the integral $A_{\alpha}^{1 j}$ in (3.2) is a particular case of the integrals on the r.h.s. of (3.3) when $k=1$. Therefore, it remains to consider the integral on the r.h.s. of the (3.3) and the integral $A_{\alpha}^{0 j}$ defined by (3.1). For both of them, an analysis shows that they are promptly expressed as linear combinations of three standard terms:

$$
\begin{gathered}
{\left[\left[y_{3}^{l+1+\alpha} \ln \left[\left(x_{2}-b_{\xi}-a_{\xi} y_{3}\right)+R_{\xi}\right]\right]_{\xi=b}^{\xi=f}\right]_{y_{3}=0}^{y_{3}=h},} \\
{\left[u_{s}^{\xi} \int_{0}^{h} \frac{y_{3}^{\alpha} y_{3}^{s}}{R_{\xi}} d y_{3}\right]_{\xi=b}^{\xi=f}}
\end{gathered}
$$




$$
\left[\int_{0}^{h} \frac{y_{3}^{\alpha}\left(\widetilde{A} y_{3}+\widetilde{B}\right)}{R_{0}^{2} R_{\xi}} d y_{3}\right]_{\xi=b}^{\xi=f}
$$

where $u_{s}^{\xi}, \widetilde{A}$ and $\widetilde{B}$ are known coefficients depending on $\xi, R_{0}^{2}=x_{1}^{2}+\left(x_{3}-y_{3}\right)^{2}$.

From (3.4)-(3.6) it follows that the problem is reduced to calculation of the integrals (3.5), (3.6) and their partial derivatives of the first, second and third order. Differentiation of (3.4), being trivial, we focus on the derivatives of the integrals (3.5) and (3.6).

\section{Main integrals defining the first, second and third derivatives of standard terms}

Evaluation of the first, second and third derivatives of the standard term (3.5) shows that it results in two new standard terms:

$$
\left[v_{i}^{\xi} \int_{0}^{h} \frac{y_{3}^{\alpha}}{\left(y_{3}+z_{\xi}\right)^{i} R_{\xi}} d y_{3}\right]_{\xi=b}^{\xi=f}, \quad\left[\bar{v}_{i}^{\xi} \int_{0}^{h} \frac{y_{3}^{\alpha}}{\left(y_{3}+\overline{z_{\xi}}\right)^{i} R_{\xi}} d y_{3}\right]_{\xi=b}^{\xi=f},
$$

where $v_{i}^{\xi}$ are known, in general complex, coefficients $(i=1,2,3)$; $z_{\xi}$ is the complex root of the polynomial $R_{\xi}^{2}$, so that $\left(1+a_{\xi}^{2}\right)\left(y_{3}+z_{\xi}\right)\left(y_{3}+\overline{z_{\xi}}\right)=R_{\xi}^{2}$; the overbar denotes complex conjugation.

Similar analysis of the partial derivatives of the standard term (3.6) also yields two new standard terms:

$$
\left[w_{j}^{\xi} \int_{0}^{h} \frac{y_{3}^{\alpha} d y_{3}}{\left(y_{3}+z_{0}\right)^{j} R_{\xi}}\right]_{\xi=b}^{\xi=f}, \quad\left[\bar{w}_{j}^{\xi} \int_{0}^{h} \frac{y_{3}^{\alpha} d y_{3}}{\left(y_{3}+\bar{z}_{0}\right)^{j} R_{\xi}}\right]_{\xi=b}^{\xi=f},
$$

where $w_{j}^{\xi}$ are known, in general complex, coefficients; $z_{0}=-x_{3}+\mathrm{i} x_{1}$ is the root of $R_{0}^{2}, j=1,2,3,4$, when $x_{1} \neq 0$; in the case $x_{1}=0$ we have $z_{0}=\bar{z}_{0}=-x_{3}$ and then $j=1,2, \ldots, 8$. Actually (4.2) are particular cases of (4.1) when the root $z_{\xi}$ of $R_{\xi}^{2}$ is changed to the root $z_{0}$ of $R_{0}^{2}$.

Noting that the second expression in (4.1) and (4.2) are the conjugated first ones, we come to the conclusion that the problem is reduced to evaluation of three types of integrals, at most:

$$
\left[u_{\xi} \int_{0}^{h} \frac{y_{3}^{\alpha} y_{3}^{s}}{R_{\xi}} d y_{3}\right]_{\xi=b}^{\xi=f},\left[v_{\xi}^{i} \int_{0}^{h} \frac{y_{3}^{\alpha}}{\left(y_{3}+z_{\xi}\right)^{i} R_{\xi}} d y_{3}\right]_{\xi=b}^{\xi=f},\left[w_{\xi}^{j} \int_{0}^{h} \frac{y_{3}^{\alpha} d y_{3}}{\left(y_{3}+z_{0}\right)^{j} R_{\xi}}\right]_{\xi=b}^{\xi=f}
$$

where $i=1,2,3, j=1,2,3,4$ for $x_{1} \neq 0$ and $j=1,2, \ldots, 8$ for $x_{1}=0$. Emphasise that when representing the exponent $\alpha$ as a proper rational fracture $\alpha=n / m,(n<m)$, 
the integrals (4.3) may be evaluated recurrently. Below we give the explicit formulae for the cases most important for application: $\alpha=0$ and $\alpha=1 / 2$. Before presenting them, we distinguish three cases which suggest simplifications.

(i) Differentiation with respect to $x_{2}$. In this case, we may avoid using the recursive equation (3.3) by the method suggested in the paper [5]. Specifically, by the relation $\partial A^{k l} / \partial x_{2}=-\partial A^{k l} / \partial y_{2}$ we obtain

$$
\frac{\partial A^{k l}}{\partial x_{2}}=-\int_{0}^{h}\left[y_{3}^{l+\alpha} \frac{\left(x_{2}-b_{\xi}-a_{\xi} y_{3}\right)^{k}}{R_{\xi}}\right]_{\xi=b}^{\xi=f} d y_{3}
$$

This shows that differentiation with respect to $x_{2}$ immediately leads to arithmetic operations with the expressions (4.1).

(ii) Differentiation with respect to $x_{1}$. By differentiating equation (3.3) with respect to $x_{1}$, we obtain:

$$
\begin{gathered}
\frac{\partial A_{\alpha}^{k l}}{\partial x_{1}}=-\frac{1}{k}\left[\int_{0}^{h} \frac{x_{1} y_{3}^{\alpha+l}\left(x_{2}-b_{\xi}-a_{\xi} y_{3}\right)^{k-1}}{R_{\xi}} d y_{3}\right]_{\xi=b}^{\xi=f}+ \\
-\frac{k-1}{k}\left(2 x_{1} A_{\alpha}^{(k-2) l}+\left(x_{1}^{2}+x_{3}^{2}\right) \frac{\partial A_{\alpha}^{(k-2) l}}{\partial x_{1}}-2 x_{3} \frac{\partial A_{\alpha}^{(k-2)(l+1)}}{\partial x_{1}}+\frac{\partial A_{\alpha}^{(k-2)(l+2)}}{\partial x_{1}}\right) .
\end{gathered}
$$

The derivative of the starting integral $\frac{\partial A_{\alpha}^{1 l}}{\partial x_{1}}$ has the form of the first integral on the right hand side of the formula (4.5). Thus it is enough to consider the derivative of the starting integral $A_{\alpha}^{0 l}$.

$$
\frac{\partial A_{\alpha}^{0 l}}{\partial x_{1}}=x_{1} \int_{0}^{h}\left[\frac{y_{3}^{l+\alpha}\left(x_{2}-b_{\xi}-a_{\xi} y_{3}\right)}{R_{0}^{2} R_{\xi}}\right]_{\xi=b}^{\xi=f} d y_{3}-x_{1} \int_{0}^{h}\left[\frac{y_{3}^{l+\alpha}}{R_{0}^{2}}\right]_{\xi=b}^{\xi=f} d y_{3} .
$$

The first integral after decomposition into a sum of real partial fractions is evaluted by arithmetic operations with the integrals (3.5) and (3.6). The second integral does not depend on $\xi$, therefore it is zero. We see that evaluation of partial derivatives, containing differentiation with respect to $x_{1}$, is reduced to evaluation of expressions of the forms (4.1) for $i=1,2$ and (4.2) for $j=1,2,3$.

(iii) Double differentiation with respect to $x_{3}$. Since the function $1 / R$ satisfies the Laplace equation when $R \neq 0$, we may avoid repeated differentiation with respect to $x_{3}$ by using the equation

$$
\frac{\partial^{2} A_{\alpha}^{k l}}{\partial x_{3}^{2}}=-\left(\frac{\partial^{2} A_{\alpha}^{k l}}{\partial x_{1}^{2}}+\frac{\partial^{2} A_{\alpha}^{k l}}{\partial x_{2}^{2}}\right) .
$$

Then simplifications of points (i) and (ii) become available. 


\section{Case of smooth density $(\alpha=0)$}

In this case, all the integrals are evaluated analytically. Specifically, the integrals (3.5), (4.1) and (4.2) become respectively:

$$
\begin{gathered}
I_{s}=\int_{0}^{h} \frac{y_{3}^{s}}{\sqrt{\left(y_{3}+z_{\xi}\right)\left(y_{3}+\overline{z_{\xi}}\right)}} d y_{3}, \quad J_{s}=\int_{0}^{h} \frac{1}{\left(y_{3}+z_{\xi}\right)^{s} \sqrt{\left(y_{3}+z_{\xi}\right)\left(y_{3}+\overline{z_{\xi}}\right)}} d y_{3}, \\
\text { and } K_{s}=\int_{0}^{h} \frac{d y_{3}}{\left(y_{3}+z_{0}\right)^{s} \sqrt{\left(y_{3}+z_{\xi}\right)\left(y_{3}+\overline{z_{\xi}}\right)}} .
\end{gathered}
$$

Each of them is evaluated recurrently, with starting expressions:

$$
\begin{aligned}
& I_{0}=J_{0}=K_{0}=2\left[\ln \left(\sqrt{y_{3}+z_{\xi}}+\sqrt{y_{3}+\overline{z_{\xi}}}\right)\right]_{y_{3}=0}^{y_{3}=h}, \\
& I_{1}=\left[\sqrt{\left(y_{3}+z_{\xi}\right)\left(y_{3}+\overline{z_{\xi}}\right)}\right]_{y_{3}=0}^{y_{3}=h}-\operatorname{Re}\left(z_{\xi}\right) I_{0}, \\
& K_{1}=\frac{2\left[\arctan \left(\frac{\sqrt{y_{3}+z_{\xi}} \sqrt{z_{\xi}-z_{0}}}{\sqrt{y_{3}+z_{\xi}} \sqrt{z_{0}-z_{\xi}}}\right)\right]_{y_{3}=0}^{y_{3}=h}}{\sqrt{z_{0}-z_{\xi}} \sqrt{\overline{z_{\xi}}-z_{0}}} .
\end{aligned}
$$

The recursive formulas are:

$$
\begin{gathered}
I_{s}=\frac{1}{s}\left(\left[y_{3}^{s-1} \sqrt{\left(y_{3}+z_{\xi}\right)\left(y_{3}+\overline{z_{\xi}}\right)}\right]_{y_{3}=0}^{y_{3}=h}-(2 s-1) \operatorname{Re}\left(z_{\xi}\right) I_{s-1}-(s-1)\left|z_{\xi}\right|^{2} I_{s-2}\right) \\
J_{s}=\frac{1}{\left(s-\frac{1}{2}\right)\left(z_{\xi}-\overline{z_{\xi}}\right)}\left(\left[\frac{\sqrt{y_{3}+z_{\xi}}}{\left(y_{3}+z_{\xi}\right)^{s-\frac{1}{2}}}\right]_{y_{3}=0}^{y_{3}=h}+(s-1) J_{s-1}\right) \\
K_{s}=\frac{1}{(s-1)\left(z_{\xi}-z_{0}\right)\left(\overline{z_{\xi}}-z_{0}\right)}\left(-\left[\frac{\sqrt{\left(y_{3}+z_{\xi}\right)\left(y_{3}+\overline{z_{\xi}}\right)}}{\left(y_{3}+z_{0}\right)^{s-1}}\right]_{y_{3}=0}^{y_{3}=h}+\right. \\
\left.+\left(s-\frac{3}{2}\right)\left(2 z_{0}-z_{\xi}-\overline{z_{\xi}}\right) K_{s-1}-(s-2) K_{s-2}\right) .
\end{gathered}
$$

Note that in the considered case, the representation of the trapezoid as a sum of right triangles and a rectangle, allows us to use also the efficient method suggested in $[5]$. 


\section{The case of the density with square-root asymp- totics near the element edge $(\alpha=1 / 2)$}

In this case, the starting integrals for evaluation of the integrals

$$
\begin{gathered}
I_{s}=\int_{0}^{h} \frac{y_{3}^{s} q d y_{3}}{\sqrt{y_{3}\left(y_{3}+z_{\xi}\right)\left(y_{3}+\overline{z_{\xi}}\right)}}, \quad J_{s}=\int_{0}^{h} \frac{d y_{3}}{\left(y_{3}+z_{\xi}\right)^{s} \sqrt{y_{3}\left(y_{3}+z_{\xi}\right)\left(y_{3}+\overline{z_{\xi}}\right)}}, \\
K_{s}=\int_{0}^{h} \frac{d y_{3}}{\sqrt{y_{3}\left(y_{3}+z_{\xi}\right)\left(y_{3}+\overline{z_{\xi}}\right)}\left(y_{3}+z_{0}\right)^{s}},
\end{gathered}
$$

are:

$$
\begin{gathered}
I_{0}=J_{0}=K_{0}=\int_{0}^{h} \frac{d y_{3}}{\sqrt{y_{3}\left(y_{3}+z_{\xi}\right)\left(y_{3}+\overline{z_{\xi}}\right)}}, \\
I_{1}=\int_{0}^{h} \frac{y_{3}}{\sqrt{y_{3}\left(y_{3}+z_{\xi}\right)\left(y_{3}+\overline{z_{\xi}}\right)}} d y_{3}, \\
J_{1}=\int_{0}^{h} \frac{d y_{3}}{\left(y_{3}+z_{\xi}\right) \sqrt{y_{3}\left(y_{3}+z_{\xi}\right)\left(y_{3}+\overline{z_{\xi}}\right)}}, \\
K_{1}=\int_{0}^{h} \frac{d y_{3}}{\sqrt{y_{3}\left(y_{3}+z_{\xi}\right)\left(y_{3}+\overline{z_{\xi}}\right)}\left(y_{3}+z_{0}\right)}, \\
K_{2}=\int_{0}^{h} \frac{d y_{3}}{\sqrt{y_{3}\left(y_{3}+z_{\xi}\right)\left(y_{3}+\overline{z_{\xi}}\right)}\left(y_{3}+z_{0}\right)^{2}} .
\end{gathered}
$$

The recursive formulae are:

$$
\begin{aligned}
I_{s}= & \frac{1}{(2 s-1)}\left(2\left[y_{3}^{s-2} \sqrt{y_{3}\left(y_{3}+z_{\xi}\right)\left(y_{3}+\overline{z_{\xi}}\right)}\right]_{y_{3}=0}^{y_{3}=h}+\right. \\
& \left.-4 \operatorname{Re}\left(z_{\xi}\right)(s-1) I_{s-1}-\left|z_{\xi}\right|^{2}(2 s-3) I_{s-2}\right), \\
J_{s}= & \frac{1}{\left(s-\frac{1}{2}\right)\left(\overline{z_{\xi}}-z_{\xi}\right) z_{\xi}}\left[\left[\frac{\sqrt{\left(y_{3}+\overline{z_{\xi}}\right) y_{3}}}{\left(y_{3}+z_{\xi}\right)^{s-\frac{1}{2}}}\right]_{y_{3}=0}^{y_{3}=h}+\right. \\
& \left.+(s-1)\left(\overline{z_{\xi}}-2 z_{\xi}\right) J_{s-1}+\left(s-\frac{3}{2}\right) J_{s-2}\right],
\end{aligned}
$$




$$
\begin{gathered}
K_{s}=\frac{1}{2(s-1)\left(z_{0}-z_{\xi}\right)\left(z_{0}-\overline{z_{\xi}}\right) z_{0}}\left(\left[\frac{2 \sqrt{y_{3}\left(y_{3}+z_{\xi}\right)\left(y_{3}+\overline{z_{\xi}}\right)}}{\left(y_{3}+z_{0}\right)^{s-1}}\right]_{y_{3}=0}^{y_{3}=h}+\right. \\
\left.+(2 s-5) K_{s-3}-2(s-2)\left(3 z_{0}-\overline{z_{\xi}}-z_{\xi}\right) K_{s-2}\right)+ \\
+\frac{(2 s-3)}{2(s-1)}\left(\frac{1}{z_{0}}+\frac{1}{z_{0}-z_{\xi}}+\frac{1}{z_{0}-\overline{z_{\xi}}}\right) K_{s-1} .
\end{gathered}
$$

Remark 6.1 For $z_{0}=0$ (i.e. $x_{1}=0, x_{3}=0$ ),

$$
K_{s}=\int_{0}^{h} \frac{d y_{3}}{\sqrt{y_{3}\left(y_{3}+z_{\xi}\right)\left(y_{3}+\overline{z_{\xi}}\right)}\left(y_{3}\right)^{s}},
$$

and the recursive formula becomes:

$$
\begin{aligned}
K_{s}=- & \frac{1}{\left(s-\frac{1}{2}\right)\left|z_{\xi}\right|^{2}}\left(\left[y_{3}^{-\left(s-\frac{1}{2}\right)} \sqrt{\left(y_{3}+z_{\xi}\right)\left(y_{3}+\overline{z_{\xi}}\right)}\right]_{y_{3}=0}^{y_{3}=h}+\right. \\
& \left.+\left(s-\frac{3}{2}\right) K_{s-2}+2 \operatorname{Re}\left(z_{\xi}\right)(s-1) K_{s-1}\right)
\end{aligned}
$$

with starting integrals:

$$
K_{-1}=I_{1}, \quad K_{0}=I_{0}
$$

Evaluation of the starting elliptic integrals $I_{0}, I_{1}, J_{1}, K_{1}$ and $K_{2}$ is efficiently performed by proper adjusting the Carlson algorithms as explained in the next section.

\section{Efficient evaluation of standard elliptic integrals for problems involving cracks $(\alpha=1 / 2)$}

The conventional methods of evaluation the elliptic integrals employ Gauss and Landen transformations [6]. They converge quadratically and work well for elliptic integrals of the first and second kind. However, as emphasised in [6] and confirmed by our experience, they suffer from lost of significant digits for the integrals of the third kind needed for our purpose. In contrast, the Carlson algorithm provides a unified method for all the three kinds of integrals with extremely high efficiency. To use this algorithm, we introduce the new variable $t$ defined by equation:

$$
y_{3}=\frac{1}{t+\frac{1}{h}} .
$$


Then the starting integrals become:

$$
\begin{gathered}
I_{0}=\frac{1}{\left|z_{\xi}\right|} \int_{0}^{\infty} \frac{d t}{\sqrt{\left(t+\frac{1}{h}\right)\left(t+\frac{1}{h}+\frac{1}{z_{\xi}}\right)\left(t+\frac{1}{h}+\frac{1}{\bar{z}_{\xi}}\right)}}, \\
I_{1}=\frac{1}{\left|z_{\xi}\right|} \int_{0}^{\infty} \frac{d t}{\left(t+\frac{1}{h}\right)^{\frac{3}{2}} \sqrt{\left(t+\frac{1}{h}+\frac{1}{z_{\xi}}\right)\left(t+\frac{1}{h}+\frac{1}{\bar{z}_{\xi}}\right)}} \\
J_{1}=\frac{1}{z_{\xi}\left|z_{\xi}\right|} \int_{0}^{\infty} \frac{\left(t+\frac{1}{h}\right) d t}{\sqrt{\left(t+\frac{1}{h}\right)\left(t+\frac{1}{h}+\frac{1}{z_{\xi}}\right)\left(t+\frac{1}{h}+\frac{1}{\bar{z}_{\xi}}\right)}\left(t+\frac{1}{h}+\frac{1}{z_{\xi}}\right)} \\
K_{1}=\frac{1}{\left|z_{\xi}\right| z_{0}} \int_{0}^{\infty} \frac{\left(t+\frac{1}{h}\right) d t}{\sqrt{\left(t+\frac{1}{h}\right)\left(t+\frac{1}{h}+\frac{1}{z_{\xi}}\right)\left(t+\frac{1}{h}+\frac{1}{\bar{z}_{\xi}}\right)}\left(t+\frac{1}{h}+\frac{1}{z_{0}}\right)} \\
K_{2}=\frac{1}{\left|z_{\xi}\right| z_{0}^{2}} \int_{0}^{\infty} \frac{\left(t+\frac{1}{h}\right)^{2} d t}{\sqrt{\left(t+\frac{1}{h}\right)\left(t+\frac{1}{h}+\frac{1}{z_{\xi}}\right)\left(t+\frac{1}{h}+\frac{1}{z_{\xi}}\right)}\left(t+\frac{1}{h}+\frac{1}{z_{0}}\right)^{2}}
\end{gathered}
$$

They are promptly expressed in terms of Carlson integrals $R_{F}, R_{D}$ and $R_{J}$ of the first, second and third kind, respectively, defined as:

$$
\begin{aligned}
R_{F}(x, y, z) & =\frac{1}{2} \int_{0}^{\infty}[(t+x)(t+y)(t+z)]^{-\frac{1}{2}} d t \\
R_{D}(z, y, z)=R_{J}(x, y, z, z) & =\frac{3}{2} \int_{0}^{\infty}[(t+x)(t+y)]^{-\frac{1}{2}}(t+z)^{-\frac{3}{2}} d t \\
R_{J}(x, y, z, p) & =\frac{3}{2} \int_{0}^{\infty}[(t+x)(t+y)(t+z)]^{-\frac{1}{2}}(t+p)^{-1} d t .
\end{aligned}
$$

In terms of the Carlson integrals, the starting integrals are:

$$
\begin{gathered}
I_{0}=\frac{2}{\left|z_{\xi}\right|} R_{F}\left(\frac{1}{h}, \frac{1}{h}+\frac{1}{z_{\xi}}, \frac{1}{h}+\frac{1}{\bar{z}_{\xi}}\right), \\
I_{1}=\frac{2}{3\left|z_{\xi}\right|} R_{D}\left(\frac{1}{h}+\frac{1}{z_{\xi}}, \frac{1}{h}+\frac{1}{\bar{z}_{\xi}}, \frac{1}{h}\right), \\
J_{1}=\frac{I_{0}}{z_{\xi}}-\frac{2}{3 z_{\xi}^{2}\left|z_{\xi}\right|} R_{D}\left(\frac{1}{h}, \frac{1}{h}+\frac{1}{\bar{z}_{\xi}}, \frac{1}{h}+\frac{1}{z_{\xi}}\right), \\
K_{1}=\frac{I_{0}}{z_{0}}-\frac{2}{3\left|z_{\xi}\right| z_{0}^{2}} R_{J}\left(\frac{1}{h}, \frac{1}{h}+\frac{1}{z_{\xi}}, \frac{1}{h}+\frac{1}{\bar{z}_{\xi}}, \frac{1}{h}+\frac{1}{z_{0}}\right),
\end{gathered}
$$




$$
\begin{gathered}
K_{2}=-\frac{I_{0}}{2 z_{0}^{2}}\left(1+\frac{z_{\xi}}{z_{0}-z_{\xi}}+\frac{\bar{z}_{\xi}}{z_{0}-\bar{z}_{\xi}}\right)-\frac{I_{1}}{2 z_{0}^{3}}+ \\
+\frac{K_{1}}{2 z_{0}}\left(3+\frac{z_{\xi}}{z_{0}-z_{\xi}}+\frac{\bar{z}_{\xi}}{z_{0}-\bar{z}_{\xi}}\right)+\frac{1}{\left|z_{\xi}\right| z_{0}^{4}} \frac{\sqrt{h}}{\left(\frac{1}{h}+\frac{1}{z_{0}}\right)\left|\frac{1}{h}+\frac{1}{z_{\xi}}\right|}+ \\
-\frac{1}{3\left|z_{\xi}\right| z_{0}^{3}}\left(\frac{z_{\xi}}{z_{\xi}-z_{0}} R_{D}\left(\frac{1}{h}, \frac{1}{h}+\frac{1}{\bar{z}_{\xi}}, \frac{1}{h}+\frac{1}{z_{\xi}}\right)+\frac{\bar{z}_{\xi}}{\bar{z}_{\xi}-z_{0}} R_{D}\left(\frac{1}{h}, \frac{1}{h}+\frac{1}{z_{\xi}}, \frac{1}{h}+\frac{1}{\bar{z}_{\xi}}\right)\right) .
\end{gathered}
$$

Finally, we need to evaluate five Carlson integrals only:

$$
\begin{array}{ll}
R_{D}\left(\frac{1}{h}+\frac{1}{z_{\xi}}, \frac{1}{h}+\frac{1}{z_{\xi}}, \frac{1}{h}\right), & R_{D}\left(\frac{1}{h}, \frac{1}{h}+\frac{1}{z_{\xi}}, \frac{1}{h}+\frac{1}{z_{\xi}}\right), \\
R_{D}\left(\frac{1}{h}, \frac{1}{h}+\frac{1}{z_{\xi}}, \frac{1}{h}+\frac{1}{z_{\xi}}\right), & R_{F}\left(\frac{1}{h}, \frac{1}{h}+\frac{1}{z_{\xi}}, \frac{1}{h}+\frac{1}{z_{\xi}},\right), \\
R_{J}\left(\frac{1}{h}, \frac{1}{h}+\frac{1}{z_{\xi}}, \frac{1}{h}+\frac{1}{z_{\xi}}, \frac{1}{h}+\frac{1}{z_{0}}\right) . &
\end{array}
$$

The integrals $R_{D}$ and $R_{F}$ are evaluated very fast and accurately by algorithms presented by Carlson in the paper [3]. The same also refers to the integrals $R_{J}$ when its last argument in not a negative real number. The case, when the last argument $\left(\frac{1}{h}+\frac{1}{z_{0}}\right)$ of the integral $R_{J}$ is a real negative number, is special. It occurs when the field point is within the strip $x_{1}=0,0<x_{3}<h$. Then the integral $R_{J}$ is a singular real Cauchy integral:

$$
R_{J}\left(\frac{1}{h}, \frac{1}{h}+\frac{1}{z_{\xi}}, \frac{1}{h}+\frac{1}{\bar{z}_{\xi}}, \frac{1}{h}+\frac{1}{z_{0}}\right)=\int_{0}^{\infty} \frac{d t}{\sqrt{t+\frac{1}{h}} \sqrt{f+g t+t^{2}}\left(t+\frac{1}{h}+\frac{1}{z_{0}}\right)},
$$

where $f=\left|z_{\xi}\right|^{2}, g=2 \operatorname{Re} z_{\xi}$. In the paper [2] Carlson provides equations serving for efficient of this integral:

$$
\begin{gathered}
R_{J}=\frac{2 c_{11}}{3 c_{44}}\left[-4 x_{3}\left(c_{14}^{2}+\sqrt{c_{11}^{2} c_{44}^{2}}\right) R_{J}\left(M^{2}, L_{-}^{2}, L_{+}^{2}, W_{+}^{2}\right)+\right. \\
\left.-6 R_{F}\left(M^{2}, L_{-}^{2}, L_{+}^{2}\right)+3 R_{C}\left(U^{2}, W^{2}\right)-2 R_{C}\left(P^{2}, Q^{2}\right)\right]
\end{gathered}
$$

where

$$
\begin{aligned}
& c_{11}^{2}=2\left(f-\frac{g}{h}+\frac{1}{h^{2}}\right) \\
& c_{14}^{2}=2\left(f-g\left(\frac{1}{h}+\frac{1}{2 z_{0}}\right)+\frac{1}{h}\left(\frac{1}{h}+\frac{1}{z_{0}}\right)\right), \\
& c_{44}^{2}=2\left(f-g\left(\frac{1}{h}+\frac{1}{z_{0}}\right)+\left(\frac{1}{h}+\frac{1}{z_{0}}\right)^{2}\right),
\end{aligned}
$$


and

$$
\begin{aligned}
M^{2} & =2 \sqrt{f}+g, \\
U^{2} & =\frac{1}{h}, \\
W^{2} & =U^{2}+\frac{1}{2} z_{0} c_{11}^{2}, \\
W_{+}^{2} & =M^{2}+z_{0}\left(c_{14}^{2}+c_{11} c_{44}\right), \\
L_{ \pm}^{2} & =M^{2}+\left(\frac{2}{h}-g\right) \pm c_{11} \sqrt{2}, \\
Q^{2} & =W^{2}\left(1+\frac{h}{z_{0}}\right), \\
P^{2} & =Q^{2}-\frac{1}{2} z_{0} c_{44}^{2}, \\
R_{C}(a, b) & =R_{F}(a, b, b) .
\end{aligned}
$$

With using these equations, evaluation of the elliptic integrals, needed for problems involving cracks, becomes extremely efficient. Our experience shows that calculations of influence coefficients for square-root edge elements $(\alpha=1 / 2)$ are performed as accurate and fast as those for ordinary elements $(\alpha=0)$.

We believe that similar, highly efficient algorithms may be developed for any proper fraction $\alpha=m / n(m<n)$.

\section{Acknowledgement}

Authors gratefully acknowledge the support of the European Research Agency (FP7PEOPLE-2009-IAPP Marie Curie IAPP transfer of knowledge programme, Project Reference \#251475).

\section{References}

[1] Banerjee P. K., Butterfield R., Boundary element methods in engineering science, McGrawHill Book Co., UK, 1981.

[2] Carlson B. C., A table of elliptic integrals: one quadratic factor, Mathematics of computation, 56, 1991, pp. 267-280.

[3] Carlson B. C., Numerical computation of real or complex elliptic integrals, Numerical Algorithms, 10, 1995, pp. 13-26.

[4] Linkov A. M., Boundary integral equations in elasticity theory, Kluwer Academic Publishers, Dordrecht-Boston-London, 2002.

[5] Linkov A. M., Zoubkov V. V. and Kheib M. A., A method of solving threedimensional problems of seam working and geological faults, J. Min. Sci., vol. 33 (4), 1997, pp. 3-18.

[6] Zill D. G., Carlson B. C., Symmetric elliptic integrals of the third kind, Mathematics of computation, 24, 1970, pp. 199-214.

DOI: $10.7862 / \mathrm{rf.2014.8}$ 
Liliana Rybarska-Rusinek - corresponding author email: rybarska@prz.edu.pl

Dawid Jaworski

email: dawidj.poczta@gmail.com

\author{
Aleksandr Linkov \\ email: linkoval@prz.edu.pl \\ Department of Mathematics, \\ Rzeszów University of Technology, \\ al. Powstańców Warszawy 12, \\ 35-959 Rzeszów, Poland \\ Received 10.10.2013, Accepted 18.12.2013
}

